\title{
ORTHOGONALITY PRESERVING PROPERTY, WIGNER EQUATION, AND STABILITY
}

\author{
JACEK CHMIELIŃSKI
}

Received 3 November 2005; Accepted 2 July 2006

We deal with the stability of the orthogonality preserving property in the class of mappings phase-equivalent to linear or conjugate-linear ones. We give a characterization of approximately orthogonality preserving mappings in this class and we show some connections between the considered stability and the stability of the Wigner equation.

Copyright (C) 2006 Jacek Chmieliński. This is an open access article distributed under the Creative Commons Attribution License, which permits unrestricted use, distribution, and reproduction in any medium, provided the original work is properly cited.

\section{Introduction}

Let $X$ and $Y$ be (real or complex) inner-product spaces (by $\mathbb{K}$ we denote the scalar field, by $\langle\cdot \mid \cdot\rangle$ and $\|\cdot\|$ the inner product and the corresponding norm, and by $\perp$ the standard orthogonality relation). A mapping $f: X \rightarrow Y$ is called an isometry if and only if $\| f(x)-$ $f(y)\|=\| x-y \|$ for all $x, y \in X$ and is called inner-product preserving if it is a solution of the orthogonality equation

$$
\langle f(x) \mid f(y)\rangle=\langle x \mid y\rangle \quad \text { for } x, y \in X
$$

One can show that $f$ satisfies (1.1) if and only if it is a linear isometry. Similarly, $f: X \rightarrow Y$ is a solution of the functional equation

$$
\langle f(x) \mid f(y)\rangle=\langle y \mid x\rangle \quad \text { for } x, y \in X
$$

if and only if $f$ is a conjugate-linear isometry, where conjugate-linear means that $f(\lambda x+$ $\mu y)=\bar{\lambda} f(x)+\bar{\mu} f(y)$ for $x, y \in X$ and $\lambda, \mu \in \mathbb{K}$. Functions $f, g: X \rightarrow Y$ are called phaseequivalent if and only if there exists a mapping $\sigma: X \rightarrow \mathbb{K}$ such that $g(x)=\sigma(x) f(x)$ and $|\sigma(x)|=1$ for each $x \in X$. Let us denote by $\mathscr{L}^{\prime}=\mathscr{L}^{\prime}(X, Y)$ the class of all mappings which are phase-equivalent to linear or conjugate-linear ones. A mapping $f: X \rightarrow Y$ which 
satisfies the condition

$$
\forall x, y \in X: x \perp y \Longrightarrow f(x) \perp f(y)
$$

will be called orthogonality preserving (o.p.). These mappings may be very irregular (cf. examples in [3]). In [5], the author proved the stability of the orthogonality preserving property in the class of linear mappings defined on finite-dimensional inner-product spaces. The main tools used in the proof of that result were a characterization of linear orthogonality preserving mappings obtained in [3] and the stability of the orthogonality equation verified in the first part of [5]. In the present paper, we are going to extend the above-mentioned result to the class $\mathscr{L}^{\prime}$.

The class of functions preserving the absolute value of the inner product has found some applications in quantum physics. In the book of Wigner [13], the class of such operators was described. Therefore the functional equation

$$
|\langle f(x) \mid f(y)\rangle|=|\langle x \mid y\rangle|, \quad \text { for } x, y \in X,
$$

is called the Wigner equation. The celebrated Wigner's theorem states that if $f$ satisfies (1.4), then it is phase-equivalent to a linear or conjugate-linear isometry (for the proof of this theorem and comments see, e.g., $[1,11])$. Conversely, it is obvious that a mapping $f$ which is phase-equivalent to a linear or conjugate-linear isometry satisfies (1.4). We will see that it is enough to assume that $f$ is phase-equivalent to a linear or conjugate-linear orthogonality preserving mapping. This was already observed by Uhlhorn [12].

\section{Approximately orthogonality preserving mappings}

For $\varepsilon \in[0,1)$, we define an $\varepsilon$-orthogonality of vectors $u$ and $v$ :

$$
u \perp^{\varepsilon} v: \Longleftrightarrow|\langle u \mid v\rangle| \leq \varepsilon\|u\|\|v\|
$$

We call a mapping $f: X \rightarrow Y$ E-orthogonality preserving ( $\varepsilon$-o.p.) or approximately orthogonality preserving (a.o.p.) if and only if it satisfies the condition

$$
\forall x, y \in X: x \perp y \Longrightarrow f(x) \perp^{\varepsilon} f(y) .
$$

Obviously, if $\varepsilon=0$, then $f$ is orthogonality preserving.

The main result of [3] states that linear a.o.p. mappings nearly satisfy the orthogonality equation.

Proposition 2.1 (see [3, Theorem 2]). Let $f: X \rightarrow Y$ be a nonzero linear mapping satisfying (2.2) with some $\varepsilon \in[0,1)$. Then there exists $\gamma>0$ such that

$$
|\langle f(x) \mid f(y)\rangle-\gamma\langle x \mid y\rangle| \leq \hat{\varepsilon} \min \{\gamma\|x\|\|y\|,\|f(x)\|\|f(y)\|\}, \quad x, y \in X,
$$

with

$$
\widehat{\varepsilon}=4 \varepsilon\left(\frac{1}{1-\varepsilon}+\sqrt{\frac{1+\varepsilon}{1-\varepsilon}}\right) .
$$


For real spaces, one can obtain even better approximation; namely with $\varepsilon /(1-\varepsilon)$ instead of $\hat{\varepsilon}$. Let us replace now the linearity of $f$ by conjugate-linearity.

Proposition 2.2. Let $f: X \rightarrow Y$ be a nonzero conjugate-linear mapping satisfying (2.2) with some $\varepsilon \in[0,1)$. Then there exists $\gamma>0$ such that

$$
|\langle f(x) \mid f(y)\rangle-\gamma\langle y \mid x\rangle| \leq \widehat{\varepsilon} \min \{\gamma\|x\|\|y\|,\|f(x) \mid\|\|f(y)\|\}, \quad x, y \in X,
$$

with $\hat{\varepsilon}$ given by (2.4).

Proof. We only sketch the proof which runs similarly like the one of Proposition 2.1. In the first part of the proof [5, Theorem 2], it is shown (and this part remains unchanged if we replace linearity of $f$ by its conjugate-linearity) that for an arbitrarily fixed $x_{0} \in$ $X \backslash\{0\}, \gamma:=\left\|f\left(x_{0}\right)\right\|^{2} /\left\|x_{0}\right\|^{2}$ and $\delta:=\sqrt{(1+\varepsilon) /(1-\varepsilon)+2 \varepsilon \sqrt{(1+\varepsilon) /(1-\varepsilon)}} \geq 1$, one has

$$
\begin{aligned}
& \left|\|f(x)\|^{2}-\gamma\|x\|^{2}\right| \leq\left(\delta^{2}-1\right) y\|x\|^{2}, \quad x \in X, \\
& \left|\|f(x)\|^{2}-\gamma\|x\|^{2}\right| \leq\left(\delta^{2}-1\right)\|f(x)\|^{2}, \quad x \in X
\end{aligned}
$$

(cf. [3, equations (10) and (12)]). Using the polarization formula, conjugate-linearity of $f$, (2.6), we obtain for all $x, y \in X$ that

$$
|\langle f(x) \mid f(y)\rangle-\gamma\langle y \mid x\rangle| \leq\left(\delta^{2}-1\right) \min \left\{\gamma\|x\|^{2}+\gamma\|y\|^{2},\|f(x)\|^{2}+\|f(y)\|^{2}\right\} .
$$

Now, suppose $x, y \in X \backslash\{0\}$ (then we have $f(x), f(y) \in Y \backslash\{0\}$ ). Applying (2.7) for vectors $x /\|x\|$ and $y /\|y\|$, we get

$$
|\langle f(x) \mid f(y)\rangle-\gamma\langle y \mid x\rangle| \leq 2\left(\delta^{2}-1\right) \gamma\|x\|\|y\| .
$$

Analogously, applying (2.7) to vectors $x /\|f(x)\|$ and $y /\|f(y)\|$, we obtain

$$
|\langle f(x) \mid f(y)\rangle-\gamma\langle y \mid x\rangle| \leq 2\left(\delta^{2}-1\right)|| f(x)|||| f(y)|| .
$$

Since $2\left(\delta^{2}-1\right)=\widehat{\varepsilon},(2.5)$ follows for all $x, y \in X \backslash\{0\}$ and trivially for $x=0$ or $y=0$.

Using the above two propositions, we obtain the following.

Theorem 2.3. Let $f: X \rightarrow Y$ be a nonzero mapping satisfying (2.2) with some $\varepsilon \in[0,1)$ and let $f \in \mathscr{L}^{\prime}$. Then there exists $\gamma>0$ such that

$$
||\langle f(x) \mid f(y)\rangle|-\gamma|\langle x \mid y\rangle|| \leq \hat{\varepsilon} \min \{\gamma\|x\|\|y\|,\|f(x)\|\|f(y)\|\}, \quad x, y \in X,
$$

with $\hat{\varepsilon}$ given by (2.4).

Proof. Let $f$ be phase-equivalent to a linear or conjugate-linear mapping $g$. It is easily visible that $g$ also satisfies (2.2). Following Propositions 2.1 and 2.2, we observe that in 
4 Orthogonality preserving property and its stability

the case $g$ is linear, $g$ satisfies (2.3) and

$$
\begin{aligned}
||\langle f(x) \mid f(y)\rangle|-\gamma|\langle x \mid y\rangle|| & =||\langle g(x) \mid g(y)\rangle|-\gamma|\langle x \mid y\rangle|| \\
& \leq|\langle g(x) \mid g(y)\rangle-\gamma\langle x \mid y\rangle| \\
& \leq \hat{\varepsilon} \min \{\gamma\|x\|\|y\|,\|f(x)\|\|f(y)\|\}
\end{aligned}
$$

and, in the case $g$ is conjugate-linear, $g$ satisfies (2.5) and

$$
\begin{aligned}
||\langle f(x) \mid f(y)\rangle|-\gamma|\langle x \mid y\rangle|| & =||\langle g(x) \mid g(y)\rangle|-\gamma|\langle y \mid x\rangle|| \\
& \leq|\langle g(x) \mid g(y)\rangle-\gamma\langle y \mid x\rangle| \\
& \leq \hat{\varepsilon} \min \{\gamma\|x\|\|y\|,\|f(x)\|\|f(y)\|\} .
\end{aligned}
$$

Taking $\varepsilon=0$ one gets the following.

Corollary 2.4. Let $f: X \rightarrow Y$ be a nonzero orthogonality preserving mapping and let $f \in$ $\mathscr{L}^{\prime}$. Then there exists $\gamma>0$ such that

$$
|\langle f(x) \mid f(y)\rangle|=\gamma|\langle x \mid y\rangle|, \quad x, y \in X
$$

Then the following corollary follows from Wigner's theorem.

Corollary 2.5. If $f \in \mathscr{L}^{\prime}$ is a nonzero orthogonality preserving mapping, then it is phaseequivalent with a linear or conjugate-linear isometry multiplied by a positive constant.

\section{Stability}

For a general information on the stability of functional equations we refer, for example, to monographs $[7,8]$ and numerous papers dealing with this subject, also in this journal (cf., e.g., $[9,10])$.

In [5], the following stability result was proved.

Proposition 3.1 (see [5, Theorem 4]). Let X, Y be inner-product spaces and let X be finitedimensional. Then, there exists a continuous function $\delta:[0,1) \rightarrow[0,+\infty)$ with the property $\lim _{\varepsilon \rightarrow 0^{+}} \delta(\varepsilon)=0$ such that for each linear $\varepsilon$-o.p. mapping $f: X \rightarrow Y$, there exists a linear o.p. mapping $T: X \rightarrow Y$ such that

$$
\|f-T\| \leq \delta(\varepsilon) \min \{\|f\|,\|T\|\}
$$

We can now extend the class of linear mappings to the class $\mathscr{L}^{\prime}$ and consider in this broader class the stability of the orthogonality preserving property. First, we prove the following.

Lemma 3.2. Let $X$ be a finite-dimensional inner-product space with an orthonormal basis $\left\{e_{1}, \ldots, e_{n}\right\}$ and let $Y$ be an arbitrary inner-product space. Then, there exists a continuous 
mapping $\eta:[0,1) \rightarrow[0, \infty)$ such that $\lim _{\varepsilon \rightarrow 0^{+}} \eta(\varepsilon)=0$ and satisfying the following property: for $f: X \rightarrow Y$ such that (with $\varepsilon \geq 0$ )

$$
\begin{gathered}
||\left|f\left(e_{i}\right)\right|^{2}-1 \mid \leq \varepsilon, \quad i=1, \ldots, n \\
\left|\left\langle f\left(e_{i}\right) \mid f\left(e_{j}\right)\right\rangle\right| \leq \varepsilon, \quad i, j=1, \ldots, n, i \neq j \\
f\left(e_{1}\right), \ldots, f\left(e_{n}\right) \quad \text { linearly independent }
\end{gathered}
$$

there exists $I: X \rightarrow Y$ - a linear isometry such that

$$
\left\|f\left(e_{i}\right)-I\left(e_{i}\right)\right\| \leq \eta(\varepsilon), \quad i=1, \ldots, n .
$$

Proof. This result is hidden in the proof of [5, Theorem 2]. Therefore we present only a sketch of the proof.

Let $f: X \rightarrow Y$ satisfy (3.2), (3.3), and (3.4). Define $Y_{0}=\operatorname{lin}\left\{f\left(e_{1}\right), \ldots, f\left(e_{n}\right)\right\} \subset Y$. Let $H_{i}:=\operatorname{lin}\left\{f\left(e_{1}\right), \ldots, f\left(e_{i-1}\right)\right\}$ for $i=2, \ldots, n$. For a subspace $V$ of $Y_{0}$ by $V^{\perp}$, we mean the orthogonal complement of $V$ in $Y_{0}$ and $P_{V} x$ denotes the orthogonal projection of $x$ onto $V$. We define a linear mapping $I: X \rightarrow Y_{0}$ by

$$
I\left(e_{1}\right):=\frac{f\left(e_{1}\right)}{\left\|f\left(e_{1}\right)\right\|}, \quad I\left(e_{i}\right):=\frac{P_{H_{i}^{\perp}} f\left(e_{i}\right)}{\left\|P_{H_{i}^{\perp}} f\left(e_{i}\right)\right\|}, \quad i=2, \ldots, n .
$$

We have $\left\langle I\left(e_{i}\right) \mid I\left(e_{j}\right)\right\rangle=\delta_{i j}$ for $i, j=1, \ldots, n$, whence $\langle I(x) \mid I(y)\rangle=\langle x \mid y\rangle$ for $x, y \in X$. It is easily seen from (3.2) that

$$
\left\|I\left(e_{1}\right)-f\left(e_{1}\right)\right\|=\left|1-\left\|f\left(e_{1}\right)\right\|\right| \leq 1-\sqrt{1-\varepsilon}=: \eta_{1}(\varepsilon) .
$$

Then one can show inductively, exactly like in the proof of [5, Theorem 2] that for $i=$ $1, \ldots, n$, there exist continuous mappings $\eta_{i}:\left[0, \varepsilon_{i}\right) \rightarrow \mathbb{R}_{+}$(with some $\varepsilon_{i}>0$ ) such that $\lim _{\varepsilon \rightarrow 0^{+}} \eta_{i}(\varepsilon)=0$,

$$
\left\|f\left(e_{i}\right)-I\left(e_{i}\right)\right\| \leq \eta_{i}(\varepsilon)
$$

and $\eta_{1}(\varepsilon) \leq \cdots \leq \eta_{n}(\varepsilon)$. Thus we have $\left\|f\left(e_{i}\right)-I\left(e_{i}\right)\right\| \leq \eta_{n}(\varepsilon)$ for $i=1, \ldots, n$ and sufficiently small $\varepsilon$ (i.e., for $\varepsilon<\varepsilon_{n}$ ). On the other hand, for arbitrary $\varepsilon$ we have, using (3.2),

$$
\left\|f\left(e_{i}\right)-I\left(e_{i}\right)\right\| \leq\left\|f\left(e_{i}\right)\right\|+\left\|I\left(e_{i}\right)\right\| \leq \sqrt{1+\varepsilon}+1=: \eta^{\prime}(\varepsilon) .
$$

Therefore, combining $\eta_{n}$ (for small $\varepsilon$ ) with $\eta^{\prime}$ (for greater), we get a mapping $\eta$ defined on the whole interval $[0,1)$, continuous, and satisfying the condition $\lim _{\mathcal{\varepsilon} \rightarrow 0^{+}} \eta(\varepsilon)=0$, for which the assertion holds.

Note that $\eta$ depends only on the dimension of $X$.

We will use the above result in the proof of our main result.

Theorem 3.3. Let $X, Y$ be inner-product spaces and let $X$ be finite-dimensional. Then, there exist an $\varepsilon_{0} \in(0,1)$ and a continuous function $\delta:\left[0, \varepsilon_{0}\right) \rightarrow[0,+\infty)$ with the property 
$\lim _{\mathcal{E} \rightarrow 0^{+}} \delta(\varepsilon)=0$ such that for each $\varepsilon$-o.p. mapping $f: X \rightarrow Y$ from $\mathscr{L}^{\prime}$, there exists an o.p. mapping $T: X \rightarrow Y$ from $\mathscr{L}^{\prime}$ such that

$$
\|f(x)-T(x)\| \leq \delta(\varepsilon) \min \{\|f(x)\|,\|T(x)\|\}, \quad x \in X
$$

Proof. Let $\operatorname{dim} X=n$ and let $\eta$ be the mapping from the assertion of Lemma 3.2. Let $\varepsilon_{0}$ be such that for $\varepsilon<\varepsilon_{0}, \widehat{\varepsilon}<1$ and $\eta(\widehat{\varepsilon}) \sqrt{n}<1$. We will show that the mapping

$$
\delta(\varepsilon):=\frac{\eta(\widehat{\varepsilon}) \sqrt{n}}{1-\eta(\widehat{\varepsilon}) \sqrt{n}}, \quad \varepsilon \in\left(0, \varepsilon_{0}\right),
$$

satisfies the assertion of the theorem.

From now on, we assume that $\varepsilon<\varepsilon_{0}$. Let $f \neq 0$ be an $\mathcal{E}$-o.p. mapping from $\mathscr{L}^{\prime}$. Thus, according to Theorem 2.3, there exists a $\gamma>0$ such that $(2.10)$ holds. Define $\langle x \mid y\rangle^{\prime}:=$ $\gamma\langle x \mid y\rangle$ for $x, y \in X$-a new inner product in $X$ (which yields a new norm $\|x\|^{\prime}=\sqrt{\gamma}\|x\|$ for $x \in X)$. Then (2.10) gives

$$
||\langle f(x) \mid f(y)\rangle|-|\langle x \mid y\rangle^{\prime}|| \leq \widehat{\varepsilon}\|x\|^{\prime}\|y\|^{\prime}, \quad x, y \in X
$$

with $\hat{\varepsilon}$ given by (2.4). Since $f \in \mathscr{L}^{\prime}$, there exist $\sigma: X \rightarrow \mathbb{K}$ with $|\sigma(x)|=1, x \in X$ and a linear or conjugate-linear mapping $g: X \rightarrow Y$ such that $f(x)=\sigma(x) g(x), x \in X$. It follows from (3.12) that

$$
||\langle g(x) \mid g(y)\rangle|-|\langle x \mid y\rangle^{\prime}|| \leq \widehat{\varepsilon}\|x\|^{\prime}\|y\|^{\prime}, \quad x, y \in X
$$

For $\left\{e_{1}, \ldots, e_{n}\right\}$ being an orthonormal basis in $X$ (with respect to the original inner product), we define $e_{i}^{\prime}:=(1 / \sqrt{\gamma}) e_{i}(i=1, \ldots, n)$ and $\left\{e_{1}^{\prime}, \ldots, e_{n}^{\prime}\right\}$ becomes an orthonormal basis with respect to $\langle\cdot \mid \cdot\rangle^{\prime}$. It is clear that $g$ satisfies (3.2) and (3.3), that is, $\mid\left\|g\left(e_{i}^{\prime}\right)\right\|^{2}-$ $1 \mid \leq \widehat{\varepsilon}$ for $i=1, \ldots, n$ and $\left|\left\langle g\left(e_{i}^{\prime}\right) \mid g\left(e_{j}^{\prime}\right)\right\rangle\right| \leq \widehat{\varepsilon}$ for $i, j=1, \ldots, n, i \neq j$. We have also (3.4): $g\left(e_{1}^{\prime}\right), \ldots, g\left(e_{n}^{\prime}\right)$ are linearly independent. Indeed, we have from (3.13) that $\|g(x)\|=0$ implies $x=0$, whence the assumption $\lambda_{1} g\left(e_{1}^{\prime}\right)+\cdots+\lambda_{n} g\left(e_{n}^{\prime}\right)=0$ and the fact that $g$ is linear or conjugate-linear would imply $\lambda_{1} e_{1}^{\prime}+\cdots+\lambda_{n} e_{n}^{\prime}=0$ or $\overline{\lambda_{1}} e_{1}^{\prime}+\cdots+\overline{\lambda_{n}} e_{n}^{\prime}=0$, respectively, a contradiction. From Lemma 3.2 (remember that $\eta$ depends only on the dimension of $X$, in particular not on the inner product), we know that there exists $I: X \rightarrow Y$ satisfying

$$
\langle I(x) \mid I(y)\rangle=\langle x \mid y\rangle^{\prime}=\gamma\langle x \mid y\rangle, \quad x, y \in X
$$

and such that

$$
\left\|g\left(e_{i}^{\prime}\right)-I\left(e_{i}^{\prime}\right)\right\| \leq \eta(\widehat{\varepsilon}), \quad i=1, \ldots, n
$$

Let us consider two possible cases. 
(1) $g$ is linear. Then we take $J=I$ and we have for an arbitrary element $x=\xi_{1} e_{1}^{\prime}+\cdots+$ $\xi_{n} e_{n}^{\prime} \in X:$

$$
\begin{aligned}
\|g(x)-J(x)\| & =\left\|\sum_{i=1}^{n} \xi_{i} g\left(e_{i}^{\prime}\right)-\sum_{i=1}^{n} \xi_{i} I\left(e_{i}^{\prime}\right)\right\| \\
& \leq \sum_{i=1}^{n}\left|\xi_{i}\right|\left\|g\left(e_{i}^{\prime}\right)-I\left(e_{i}^{\prime}\right)\right\| \leq \eta(\widehat{\varepsilon}) \sum_{i=1}^{n}\left|\xi_{i}\right| \\
& \leq \eta(\widehat{\varepsilon}) \sqrt{n}\|x\|^{\prime}=\eta(\widehat{\varepsilon}) \sqrt{n}\|I(x)\|=\eta(\widehat{\varepsilon}) \sqrt{n}\|J(x)\| .
\end{aligned}
$$

(2) $g$ is conjugate-linear. Then we take $J(x):=\overline{\xi_{1}} I\left(e_{1}^{\prime}\right)+\cdots+\overline{\xi_{n}} I\left(e_{n}^{\prime}\right)$ for $x=\xi_{1} e_{1}^{\prime}+\cdots+$ $\xi_{n} e_{n}^{\prime} \in X$. Note that $\langle J(x) \mid J(y)\rangle=\overline{\langle x \mid y\rangle^{\prime}}$ for $x, y \in X$ whence, in particular, $\|J(x)\|=$ $\|x\|^{\prime}=\|I(x)\|, x \in X$. We have also

$$
\begin{aligned}
\|g(x)-J(x)\| & =\left\|\sum_{i=1}^{n} \bar{\xi}_{i} g\left(e_{i}^{\prime}\right)-\sum_{i=1}^{n} \bar{\xi}_{i} I\left(e_{i}^{\prime}\right)\right\| \\
& \leq \sum_{i=1}^{n}\left|\bar{\xi}_{i}\right|\left\|g\left(e_{i}^{\prime}\right)-I\left(e_{i}^{\prime}\right)\right\| \leq \eta(\widehat{\varepsilon}) \sum_{i=1}^{n}\left|\xi_{i}\right| \\
& \leq \eta(\widehat{\varepsilon}) \sqrt{n}\|x\|^{\prime}=\eta(\widehat{\varepsilon}) \sqrt{n}\|I(x)\|=\eta(\widehat{\varepsilon}) \sqrt{n}\|J(x)\| .
\end{aligned}
$$

Thus, in both cases $\|g(x)-J(x)\| \leq \eta(\widehat{\varepsilon}) \sqrt{n}\|J(x)\|$ for $x \in X$. Now we define for $x \in X$ that $T(x):=\sigma(x) J(x)$. We have then

$$
|\langle T(x) \mid T(y)\rangle|=|\langle J(x) \mid J(y)\rangle|=\left|\langle x \mid y\rangle^{\prime}\right|=\gamma|\langle x \mid y\rangle| .
$$

This shows that $T$ is an o.p. mapping and, obviously, $T \in \mathscr{L}^{\prime}$. We have also for $x \in X$ that

$$
\begin{aligned}
\|f(x)-T(x)\| & =\|\sigma(x) g(x)-\sigma(x) J(x)\|=\|g(x)-J(x)\| \\
& \leq \eta(\widehat{\varepsilon}) \sqrt{n}\|J(x)\|=\eta(\widehat{\varepsilon}) \sqrt{n}\|T(x)\| .
\end{aligned}
$$

Define $\delta^{\prime}(\varepsilon):=\eta(\widehat{\varepsilon}) \sqrt{n}$. Then we have $\|f(x)-T(x)\| \leq \delta^{\prime}(\varepsilon)\|T(x)\|$ for $x \in X$. But we have also $\|T(x)\|-\|f(x)\| \leq\|T(x)-f(x)\| \leq \delta^{\prime}(\varepsilon)\|T(x)\|$, whence (since $\delta^{\prime}(\varepsilon)<1$ for $\left.\varepsilon<\varepsilon_{0}\right)$

$$
\|f(x)-T(x)\| \leq \frac{\delta^{\prime}(\varepsilon)}{1-\delta^{\prime}(\varepsilon)}\|f(x)\| .
$$

Putting $\delta(\varepsilon):=\delta^{\prime}(\varepsilon) /\left(1-\delta^{\prime}(\varepsilon)\right)>\delta^{\prime}(\varepsilon)\left(\right.$ for $\left.\varepsilon<\varepsilon_{0}\right)$, we get

$$
\|f(x)-T(x)\| \leq \delta(\varepsilon) \min \{\|f(x)\|,\|T(x)\|\}, \quad x \in X
$$

We reformulate the above theorem, emphasizing the stability property.

Corollary 3.4. Let $X, Y$ be inner-product spaces and let $X$ be finite-dimensional. Then, for an arbitrary $\delta>0$, there exists an $\varepsilon>0$ such that each $\mathcal{\varepsilon}$-o.p. mapping $f: X \rightarrow Y$ from 
the class $\mathscr{L}^{\prime}$ can be $\delta$-approximated by an o.p. mapping $T: X \rightarrow Y$ from $\mathscr{L}^{\prime}$, that is,

$$
\|f(x)-T(x)\| \leq \delta \min \{\|f(x)\|,\|T(x)\|\}, \quad x \in X .
$$

\section{Final remarks}

Note that tracing the proof of Theorem 3.3, one can also prove the stability of the Wigner equation under the assumption that $X$ is finite-dimensional and all the considered mappings are in the class $\mathscr{L}^{\prime}$. Namely, one can show that if $f \in \mathscr{L}^{\prime}$ and satisfies the inequality

$$
||\langle f(x) \mid f(y)\rangle|-|\langle x \mid y\rangle|| \leq \varepsilon\|x\|\|y\|, \quad x, y \in X,
$$

then there exists an exact solution $T: X \rightarrow Y$ of the Wigner equation such that

$$
\|f(x)-T(x)\| \leq \delta(\varepsilon)\|x\|, \quad x \in X
$$

where $\delta$ is a continuous mapping depending on the dimension of $X$ only and satisfying the condition $\lim _{\varepsilon \rightarrow 0} \delta(\varepsilon)=0$. We can make a conjecture that the above statement holds true without assuming that $f \in \mathscr{L}^{\prime}$ (as it has been proved for the orthogonality equation in [5, Theorem 2]). In the simplest case, when $\operatorname{dim} X=1$, it has been proved in [2, Proposition 2, Theorem 3]. It is also true in the case $X=Y=\mathbb{R}^{2}$ (see [4]). On the other hand, if it were true that the Wigner equation is stable (in the considered sense) in general, we would be able to extend Theorem 3.3 on arbitrary Hilbert spaces.

Note that the stability of the Wigner equation has been proved, for $X$ and $Y$ being arbitrary Hilbert spaces, in the case where the approximate solutions are given as the solutions of the inequality

$$
||\langle f(x) \mid f(y)\rangle|-|\langle x \mid y\rangle|| \leq \varepsilon\|x\|^{p}\|y\|^{p}, \quad x \in X_{p},
$$

with $p \neq 1\left(X_{p}=X\right.$ for $p \geq 0$ and $X_{p}=X \backslash\{0\}$ for $\left.p<0\right)$. Then, one can show (cf. [2]) that there exists an exact solution $T$ of the Wigner equation such that

$$
\|f(x)-T(x)\| \leq \sqrt{\varepsilon}\|x\|^{p}, \quad x \in X_{p} .
$$

Moreover, we have the superstability in the case $X=Y=\mathbb{R}^{n}$, that is, all solutions of (4.3) are in fact exact solutions of the Wigner equation. The case $p=1$ remains, generally, unsolved. We refer also to a survey [6] devoted to various aspects of the stability of the orthogonality and Wigner equations.

\section{References}

[1] D. F. Almeida and C. S. Sharma, The first mathematical proof of Wigner's theorem, Journal of Natural Geometry 2 (1992), no. 2, 113-123.

[2] J. Chmieliński, On a singular case in the Hyers-Ulam-Rassias stability of the Wigner equation, Journal of Mathematical Analysis and Applications 289 (2004), no. 2, 571-583.

[3] __ Linear mappings approximately preserving orthogonality, Journal of Mathematical Analysis and Applications 304 (2005), no. 1, 158-169.

[4] Stability of angle-preserving mappings on the plane, Mathematical Inequalities \& Applications 8 (2005), no. 3, 497-503. 
[5] Stability of the orthogonality preserving property in finite-dimensional inner product spaces, Journal of Mathematical Analysis and Applications 318 (2006), no. 2, 433-443.

[6] Stability of the Wigner equation and related topics, to appear in Nonlinear Functional Analysis and Applications, special issue dedicated to the memory of D. H. Hyers.

[7] D. H. Hyers, G. Isac, and T. M. Rassias, Stability of Functional Equations in Several Variables, Progress in Nonlinear Differential Equations and Their Applications, vol. 34, Birkhäuser Boston, Massachusetts, 1998.

[8] S.-M. Jung, Hyers-Ulam-Rassias Stability of Functional Equations in Mathematical Analysis, Hadronic Press, Florida, 2001.

[9] _ Hyers-Ulam stability of Butler-Rassias functional equation, Journal of Inequalities and Applications 2005 (2005), no. 1, 41-47.

[10] T. Miura, S.-E. Takahasi, and G. Hirasawa, Hyers-Ulam-Rassias stability of Jordan homomorphisms on Banach algebras, Journal of Inequalities and Applications 2005 (2005), no. 4, 435441.

[11] J. Rätz, On Wigner's theorem: remarks, complements, comments, and corollaries, Aequationes Mathematicae 52 (1996), no. 1-2, 1-9.

[12] U. Uhlhorn, Representation of symmetry transformations in quantum mechanics, Arkiv Fysik 23 (1963), 307-340.

[13] E. P. Wigner, Gruppentheorie und ihre Anwendungen auf die Quantenmechanik der Atomspektren, Friedrich Vieweg und Sohn Akt.-Ges., Braunschweig, 1931.

Jacek Chmieliński: Instytut Matematyki, Akademia Pedagogiczna w Krakowie, Podchorążych 2, 30-084 Kraków, Poland

E-mail address: jacek@ap.krakow.pl 